\title{
Poorly Differentiated Small-Cell-Type Neuroendocrine Carcinoma of the Prostate: A Case Report and Literature Review
}

\author{
Kishore Kumar ${ }^{a, b} \quad$ Rafeeq Ahmed ${ }^{a, b} \quad$ Chime Chukwunonso ${ }^{a, b}$ \\ Hassan Tariq ${ }^{a, b}$ Masooma Niazic Jasbir Makker ${ }^{a, b}$ Ariyo Ihimoyan ${ }^{a, b}$
}

aDepartment of Medicine, Bronx Lebanon Hospital Center, Bronx, NY, USA;

bDivision of Gastroenterology, Bronx Lebanon Hospital Center, Bronx, NY, USA;

'Department of Pathology, Bronx Lebanon Hospital Center, Bronx, NY, USA

\section{Keywords}

Poorly differentiated small-cell-type neuroendocrine tumor - Prostate neuroendocrine tumor $\cdot$ Cisplatin/carboplatin + etoposide $\cdot$ Poor prognosis

\begin{abstract}
Neuroendocrine cells are widespread throughout the body and can give rise of neuroendocrine tumors due to abnormal growth of the chromaffin cells. Neuroendocrine tumors divide into many subtypes based on tumor grade (Ki-67 index and mitotic count) and differentiation. These tumors can be further divided into secretory and nonsecretory types based on the production of peptide hormone by tumor cells. Poorly differentiated small-cell-type neuroendocrine tumors are one of the subtypes of neuroendocrine tumors. These tumors are less common; however, they tend to be locally invasive and aggressive in behavior with poor overall median survival. Treatment of the nonsecretory small-cell type is modeled to small-cell lung
\end{abstract}




\section{Case Reports in Oncology}

Case Rep Oncol 2018;11:676-681

DOI: $10.1159 / 000493255$

2018 The Author(s). Published by S. Karger AG, Basel www.karger.com/cro

Kumar et al.: Poorly Differentiated Small-Cell-Type Neuroendocrine Carcinoma of the Prostate: A Case Report and Literature Review

cancer with a regimen consisting of platinum-based chemotherapy and etoposide with variable response. Here, we present a case of poorly differentiated small-cell neuroendocrine tumor originating from the prostate.

(C) 2018 The Author(s)

Published by S. Karger AG, Basel

\section{Introduction}

Prostatic small-cell carcinoma (PSCC) is a rare tumor that accounts for $0.5-2 \%$ of all cases of prostate cancer. The mean age at diagnosis is in the 60s. PSCC is a very aggressive tumor and has been associated with castrate-resistant metastatic prostate cancer and low prostatespecific antigen (PSA) levels, making it challenging to diagnose unless high clinical suspicion or radiologic clue is present. As adenocarcinoma of the prostate, the early manifestation of PSCC includes obstructive voiding symptoms, hematuria, symptoms related to metastatic disease, or paraneoplastic syndrome. It is noteworthy to mention that up to $33 \%$ of the cases of PSCC are diagnosed in patients with a previous history of adenocarcinoma of the prostate who may have undergone a treatment in the form of androgen deprivation or radiation therapy. We present a case of a 57-year-old Hispanic male who had undergone prostatectomy many years ago due to prostate adenocarcinoma and was now diagnosed with poorly differentiated small-cell-type adenocarcinoma of prostate.

\section{Case Presentation}

A 57-year-old male presented to the emergency department with complaints of dysuria, a decreased urinary stream, and hesitancy for 2-3 months associated with fever and chills. Review of the system was positive for chronic back pain, decreased appetite, and weight loss of 35 pounds in the past 3 months. He had no symptoms of nausea, vomiting, hematuria, saddle anesthesia, paresthesia, and lower extremity weakness. The patient had a medical history of gout, diabetes mellitus, hypertension, dyslipidemia, prostate cancer status postrobotic prostatectomy 12 years ago, and radiation therapy. He also had a history of coronary artery disease for which he underwent coronary artery bypass graft 7 years ago. His family history is significant for prostate cancer in his grandfather. He does not smoke cigarettes or use illicit drugs. He drinks alcohol socially. On arrival at the emergency room, his vitals were temperature $100.4^{\circ} \mathrm{F}$, heart rate $118 \mathrm{bpm}$, respiratory rate $20 / \mathrm{min}$, blood pressure $138 / 64 \mathrm{~mm} \mathrm{Hg}$, and he was saturating at $98 \%$ on room air. Physical examination was unremarkable except a healed sternotomy scar and mild suprapubic tenderness. Initial workup showed acute kidney injury with serum creatinine of $3.6 \mathrm{mg} / \mathrm{dL}$. Urine analysis showed pyuria and bacteriuria. The patient had recurrent fever. Septic workup was performed, and he was empirically treated for urinary tract infection with ceftriaxone. Magnetic resonance imaging of the spine showed no evidence of cord compression. Computed tomography of the abdomen without contrast showed a large lobulated prostate suspicious for neoplasm with invasion of the urinary bladder causing bilateral obstructive uropathy. Interestingly, his PSA level reported as 0.01 . He underwent nephrostomy tube placement by an interventional radiologist followed by transurethral resection of the prostate and cystoscopy with bladder mass biopsy. Histopathology 


\section{Case Reports in Oncology}

Case Rep Oncol 2018;11:676-68

DOI: $10.1159 / 000493255$

2018 The Author(s). Published by S. Karger AG, Basel www.karger.com/cro

Kumar et al.: Poorly Differentiated Small-Cell-Type Neuroendocrine Carcinoma of the Prostate: A Case Report and Literature Review

of the bladder mass showed only necrotic tissue with no viable tumor cells, and histopathology of the prostate biopsy showed small-cell-type neuroendocrine carcinoma. Immunohistochemical staining of the prostate biopsy showed that tumor cells were positive for AE1/AE3, synaptophysin, and chromogranin-A (Fig. 1a, b, c). The prostate tumor cells were negative for LCA (leukocyte common antigen), CK7 (cytokeratin 7), CK20 (cytokeratin 20), PSA, CD56 (cluster of differentiation 56), and NSE (neuron-specific enolase) antibodies, consistent with a diagnosis of small-cell-type neuroendocrine tumor of the prostate. The ki67 index was approximately $90 \%$. Metastatic workup was negative. However, diagnosis of a locally advanced neuroendocrine tumor of the prostate was made. The patient was started on palliative chemotherapy with carboplatin-etoposide every 3 weeks. However, after 2 cycles of this chemotherapy regimen, his disease complicated with colovesicle fistula formation due to locally advance malignancy, which required a diverting loop colostomy.

\section{Discussion}

In 2016, WHO reclassified neuroendocrine tumors of the prostate into 6 categories as follows: adenocarcinoma with neuroendocrine differentiation with 2 subtypes (focal and diffuse); adenocarcinoma with Paneth cell-like neuroendocrine differentiation; well-differentiated neuroendocrine tumor (carcinoid tumor); small-cell neuroendocrine carcinoma; and large-cell neuroendocrine carcinoma. Small-cell neuroendocrine carcinoma is a rare highgrade epithelial neoplasm of the prostate. The histologic features of SCC of the prostate include small tumor cells with minimum cytoplasm, indistinct cell borders, nuclear molding, fine "saltand-pepper" chromatin, lack of prominent nucleoli, extensive tumor necrosis, apoptosis, a high mitotic rate, and nuclear fragility [1]. Pathogenesis involves P53 mutation; especially with the loss of wild-type P53 it has been identified frequently. Other genetic abnormalities implicated specifically to castrate-resistant small-cell neuroendocrine tumor of the prostate is the TMPRSS2-ERG gene. Additional gene mutations that have been described in the literature include Aurora kinase A (AURKA), the oncogene $N-M Y C$, and the RB1 gene. The loss of $R B 1$ could lead to the development of PSCC and small-cell carcinoma of the lung. Simon et al. [2] reported that CD44, a glycoprotein which mediates cell-cell and cell-matrix adhesion, is expressed in $100 \%$ of the PSCC cases, and Lapuk et al. [3] reported that downregulation of REST (RE1 silencing transcription factor) resulted in the upregulation of PSCC and mixed prostatic adenocarcinoma with PSCC. Studies have suggested that CD56 expression is noted in $83-92 \%$ of the patients with PSCC. The CD44 and CD56 may act as therapeutic targets in the future treatment of PSCC. Studies have suggested that primary PSCC cases are frequently lacking androgen receptor expression, PSA, and ERG (ETS-related gene), while PSCC in the setting of castrate-resistant prostate cancer repeatedly expresses androgen receptor, PSA, and $E R G$ [4] (Table 1).

\section{Treatment}

Similarly to small cell lung cancer, PSCC is also treated with a regimen consisting of cisplatin and etoposide or carboplatin and docetaxel. Many authors have suggested to continue androgen deprivation therapy during chemotherapy for PSCC due to the concomitant presence of mixed tumors and the potential of worsening when not addressing the associated 


\section{Case Reports in Oncology}

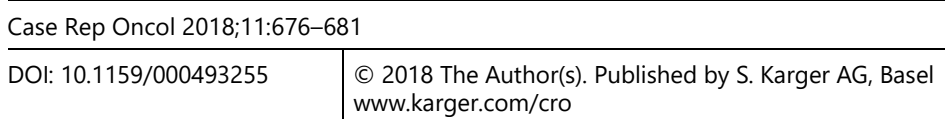
www.karger.com/cro

Kumar et al.: Poorly Differentiated Small-Cell-Type Neuroendocrine Carcinoma of the Prostate: A Case Report and Literature Review

adenocarcinoma. Local control of the PSCC has significant advantage in the overall survival of PSCC patients, either by external beam radiation therapy or radical prostatectomy. Palmgren et al. [5] reported that chemotherapy with radiation provides the best survival benefit. PSCC should be considered in patients with a low serum PSA level, inadequate response to hormonal therapy, poor differentiation of tumor cells on histology, and rapidly metastasizing disease. PSCC is an aggressive variety of prostate cancer with a median survival time following diagnosis of $\sim 10$ months, depending on the disease stage. However, $<5 \%$ survive for 2 years [6].

\section{Conclusion}

As small-cell neuroendocrine carcinoma elsewhere in the body, PSC neuroendocrine carcinoma shares many common attributes, including disease rarity, locally invasive disease, advanced stage at the time of diagnosis, prevalence in the 5th or 6th decade of life, aggressiveness, visceral metastasis, and inoperability in some cases. Typically treated with cisplatin or carboplatin and etoposide-based regimen with or without radiation, however, it exhibits poor prognosis in most cases among the many other similar features. Nonetheless, management cannot be generalized, and each case should be approached individually.

\section{Statement of Ethics}

No approval from the ethics committee had to be obtained.

\section{Disclosure Statement}

The authors have no conflicts of interest to disclose.

\section{Funding Sources}

The authors did not receive any funding.

\section{Author Contributions}

All authors contributed to the writing and review of the manuscript. 


\section{Case Reports in Oncology}

Case Rep Oncol 2018;11:676-681

DOI: $10.1159 / 000493255$

(c) 2018 The Author(s). Published by S. Karger AG, Basel www.karger.com/cro

Kumar et al.: Poorly Differentiated Small-Cell-Type Neuroendocrine Carcinoma of the Prostate: A Case Report and Literature Review

\section{References}

1 Parimi V, Goyal R, Poropatich K, Yang XJ. Neuroendocrine differentiation of prostate cancer: a review. Am J Clin Exp Urol. 2014;2(4):273-85.

2 Simon RA, di Sant'Agnese PA, Huang LS, Xu H, Yao JL, Yang Q et al. CD44 expression is a feature of prostatic small cell carcinoma and distinguishes it from its mimickers. Hum Pathol. 2009 Feb;40(2):252-8.

3 Lapuk AV, Wu C, Wyatt AW, McPherson A, McConeghy BJ, Brahmbhatt S, et al. From sequence to molecular pathology, and a mechanism driving the neuroendocrine phenotype in prostate cancer. J Pathol. 2012 Jul;227(3):286-97.

4 Monn MF, Cheng L. Emerging trends in the evaluation and management of small cell prostate cancer: a clinical and molecular perspective. Expert Rev Anticancer Ther. 2016 Oct;16(10):1029-37.

5 Palmgren JS, Karavadia SS, Wakefield MR. Unusual and underappreciated: small cell carcinoma of the prostate. Semin Oncol. 2007 Feb;34(1):22-9.

6 Alves D, Calmeiro ME, Silva R, Coelho H. Small-cell neuroendocrine cancer of the prostate: an atypical presentation of a common disease. BMJ Case Rep. 2016 Oct;2016:bcr2016216199.

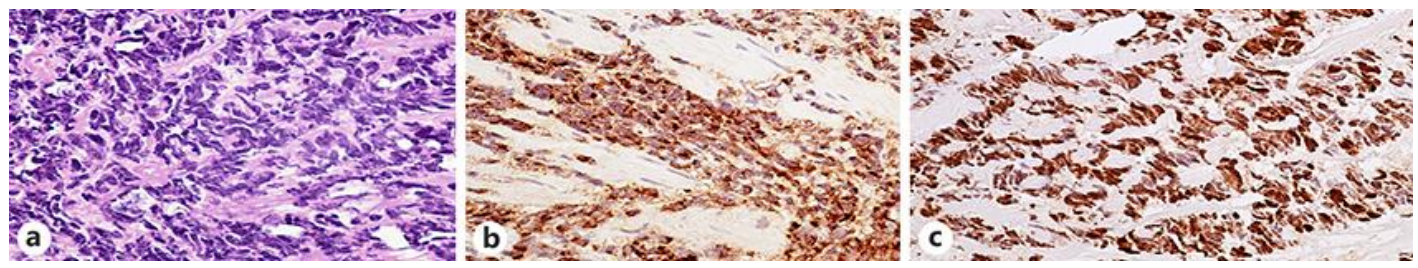

Fig. 1. a Small-cell-type neuroendocrine carcinoma. The tumor cells are oval to spindle-shaped. The cells are arranged in ribbons. HE. $\times 40$. $\mathbf{b}$ The tumor cells are immunoreactive to the neuroendocrine marker chromogranin. Immunohistochemical stain magnification, $\times 40 . c$ The tumor cells show strong intranuclear positivity to ki-67 antibodies ( $>90 \%$ ). Immunohistochemical stain magnification, $\times 340$. 
Table 1. Common molecular alteration in the pathogenesis of small-cell neuroendocrine cancer of the prostate

Tumor suppressors

RB1 protein/allelic loss (85-96\%)

TP53 allelic loss/mutation (60-100\%)

PTEN protein/allelic loss (29-63\%)

Prostate luminal epithelial markers

AR protein loss $(83-100 \%)$

PSA protein loss (81-100\%)

P501s protein loss (72\%)

Neuroendocrine elements and polypeptide hormones

Chromogranin A and/or synpatophysin and/or CD56 protein expression (92-100\%)

Bombesin protein expression (88\%)

Neural progenitor transcription factors

MYCN amplification (40-86\%)

ASCL1 protein expression

Cell cycle/mitosis markers

AURKA amplification/protein expression ${ }^{\mathrm{a}}(40-86 \%)$

UBE2C protein expression (96\%)

Genomic alterations

Increased number of genomic amplifications and deletions

TMPRSS2:-ERG gene rearrangements (46-86\%)

AR, androgen receptor; ASCL1, achaete-scute homolog 1. a AURKA and MYCN amplification are concurrent in $90 \%$ of the reported cases. 